(C2020 IEEE. Personal use of this material is permitted. Permission from IEEE must be obtained for all other uses, in any current or future media, including reprinting/republishing this material for advertising or promotional purposes, creating new collective works, for resale or redistribution to servers or lists, or reuse of any copyrighted component of this work in other works. This is the author's version of an article that has been published in the conference proceedings. The final version of record is available at https://doi.org/10.1109/PLANS46316.2020.9110139 


\title{
Matching Maps of Physical and Virtual Radio Transmitters Using Visibility Regions
}

\author{
Markus Ulmschneider, Christian Gentner and Armin Dammann \\ German Aerospace Center (DLR), Institute of Communications and Navigation \\ Muenchner Str. 20, 82334 Wessling, Germany \\ \{markus.ulmschneider,christian.gentner,armin.dammann\}@dlr.de
}

\begin{abstract}
Channel-SLAM is a multipath assisted positioning algorithm that treats multipath components as line-of-sight (LoS) signals from virtual transmitters. It maps the physical and virtual transmitters' locations simultaneously with estimating the user position with simultaneous localization and mapping (SLAM). Since Channel-SLAM is a relative localization system, the coordinate systems of transmitter maps from different users are subject to an unknown relative rotation and translation. In this paper, we present a new algorithm to estimate this rotation and translation, which we call map matching. Map matching is a requirement for collaborative Channel-SLAM, where users share transmitter maps to improve their positioning performance. Our idea is to augment maps of transmitter locations in ChannelSLAM with knowledge on from which locations there is a LoS condition to a transmitter in order to increase the robustness of map matching. We evaluate our algorithm by simulations in an indoor scenario.
\end{abstract}

Index Terms-Channel-SLAM, cooperative positioning, map matching, simultaneous localization and mapping

\section{INTRODUCTION}

Multipath propagation has universally been considered harmful for wireless localization algorithms that are based on the estimation of radio signal parameters such as phase, time of arrival (ToA) or angle of arrival (AoA). To cope with multipath propagation, standard approaches try to estimate the channel impulse response in order to remove the influence of multipath components (MPCs) on the line-of-sight (LoS) component of the received signal. Contrarily, in multipath assisted positioning, multipath propagation is regarded as a source of information that is exploited for positioning.

In the past years, various multipath assisted positioning approaches have emerged for different scenarios and technologies [1]-[5]. With Channel-SLAM [6], [7], we have introduced an algorithm that treats MPCs as LoS signals from virtual transmitters. The locations of both the virtual transmitters and the physical transmitter(s) are in general unknown. However, they can be estimated jointly with the user position with simultaneous localization and mapping (SLAM) [8]. A user hence estimates their own position and creates a map of locations of physical and virtual transmitters.

In certain scenarios such as malls, museums or public buildings, where many users require a positioning service, maps of estimated transmitter locations can be shared. In this sense, users cooperate by exchanging information on the scenario. Though, a user does in general not have any information on their position on a global scale if they do not know their initial position and heading. Instead, they create a local coordinate system in which they estimate their state jointly with the transmitter states. Thus, Channel-SLAM is only a relative positioning system. When a user shares a transmitter map with another user, the coordinate system of the shared map and the coordinate system of the user receiving the map are subject to an unknown rotation and translation [9]. The rotation and translation parameters need to be estimated to be able to exploit the information in a shared map.

We denote a set of transmitter states estimated by a user by the term user map, and the map that this user receives by the term prior map. Estimating the rotation and translation parameters relating the coordinate systems of the two maps and finding correspondences among transmitters within the two maps is denoted by the term map matching.

Map matching is particularly difficult if only one physical transmitter is present in the scenario. In such a case, the physical and virtual transmitters lack diversity as they cannot be distinguished based on their ID, the content of their signal, such as the transmitted data, or transmit time and frequency, for example. We have previously presented a paper with a reliable map matching algorithm for Channel-SLAM based on the estimated locations of transmitters in the user and the prior map [10]. The algorithm is based on a variant of the random sample consensus (RANSAC) algorithm [11], which is a widespread tool, e.g. in image registration.

Though, we have observed that in scenarios with certain geometries, map matching ambiguities may still arise. The fundamental problem is the lack of diversity among virtual transmitters, making it hard to differentiate among them. The ambiguities result in wrong estimates for the correspondences among transmitters in the two maps and thus in strongly biased estimates for the rotation and translation parameters. As a result, the user position estimate may become biased, and the filter may even diverge.

The goal of this paper is to increase the robustness of our map matching algorithm for Channel-SLAM by increasing the diversity among transmitters. A higher transmitter diversity can be achieved by exploiting the information on from where transmitters are visible. We say that a transmitter is visible to a user, if a signal from a transmitter is received by a user carrying a receiver in a $\operatorname{LoS}$ condition. Information on the locations from which a transmitter is visible or not can be mapped by users as they travel through a scenario, and it can 


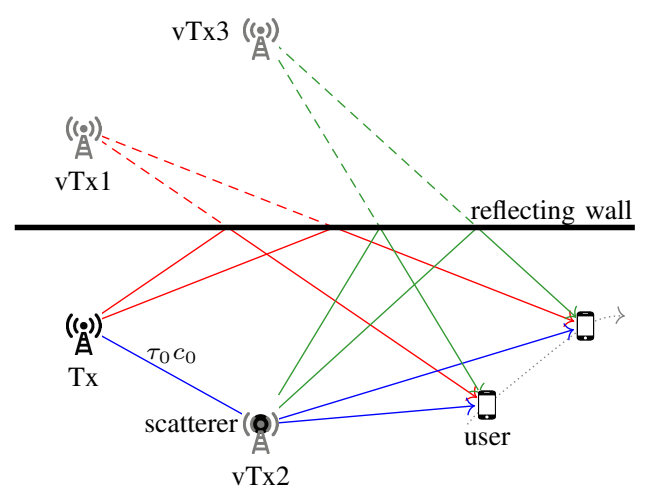

Fig. 1. The physical transmitter Tx transmits a signal that arrives at the user via three different propagation paths. On its first path drawn in red, the signal is reflected by the wall, and the corresponding signal component is regarded a LoS signal from the virtual transmitter vTx1. On the second path in blue, the signal is scattered at a point scatterer. The signal component arriving at the user is regarded a LoS signal from the virtual transmitter vTx2, which is located at the point scatterer's location. On the third propagation path drawn in green, the signal is first scattered at the point scatterer and then reflected at the wall. The corresponding virtual transmitter vTx 3 is at the scatterer location mirrored at the wall.

help in distinguishing among the various transmitters.

The remainder of the paper is organized as follows. Section III introduces multipath assisted positioning and the Channel-SLAM algorithm. Visibility maps are presented in Section III] In Section IV] we introduce our new map matching algorithm. After the simulation results in Section V. Section VI concludes the paper.

\section{Multipath Assisted Positioning And CHANNEL-SLAM}

\section{A. The Principle of Multipath Assisted Positioning}

The idea of multipath assisted positioning is exemplarily depicted in Fig. 1. The physical transmitter Tx broadcasts a signal that arrives at the user via different propagation paths. For the sake of clarity, the LoS path is omitted.

The first propagation path is drawn in red. The signal is reflected at the wall, representing a planar surface. In multipath assisted positioning, the user interprets the corresponding MPC as a LoS signal from the virtual transmitter vTx1, which is located at the location of the physical transmitter mirrored at the reflecting wall. The location of vTx1 is independent from the user position and thus static. Geometrical considerations show that the transmitters Tx and vTx1 are perfectly time synchronized.

On its second propagation path in blue, the signal is scattered at the point scatterer. The user again regards the arriving MPC as a LoS signal, transmitted by the virtual transmitter vTx2, whose location coincides with the scatterer's location. The location of $\mathrm{vTx} 2$ is thus static as well. However, there is a delay offset $\tau_{0}$ between Tx and vTx2. The delay offset corresponds to the Euclidean distance $\tau_{0} c_{0}$ between the two, where $c_{0}$ is the speed of light. The delay offset can be regarded as a clock offset.
The third propagation path in green combines the above two cases. The signal is first scattered and then reflected at the wall. The corresponding MPC arriving at the user is interpreted as a LoS signal from the virtual transmitter vTx3. In accordance with the above two cases, vTx 3 is located at the scatterer location mirrored at the wall and has a delay offset $\tau_{0}$. Any sequence of reflections and scattering can be described likewise.

\section{B. The Channel-SLAM Algorithm}

In Channel-SLAM, a single user equipped with a receiver moves through a static multipath scenario. In the scenario, there is one physical transmitter. We consider a time variant, linear multipath channel. The time variance of the channel is only due to the movement of the user. The transmit signal is denoted by $s(t)$. The received signal at the user is a superposition of signal components. We describe the $j^{\text {th }}$ signal component at time $t$ by a complex amplitude $a_{j}(t)$ and a delay, or ToA, $\tau_{j}(t)$. The received signal at time $t$ at the receiver is described as

$$
y(\tau, t)=\sum_{j} a_{j}(t) s\left(\tau-\tau_{j}(t)\right)+n(\tau)
$$

where $n(\tau)$ is colored noise that incorporates additive white Gaussian noise and dense multipath components. The receiver records a snapshot of the received signal at time instants $k$. We assume that the channel is constant for the length of one snapshot.

Channel-SLAM is a two-step approach. In a first step, a channel estimator estimates the parameters of signal components and tracks them over time based on the received signal. Within this paper, we use the Kalman Enhanced Super Resolution Tracking (KEST) estimator, which works in two stages. An inner stage is based on a snapshot-based algorithm such as the Space-Alternating Generalized ExpectationMaximization (SAGE), which estimates the parameters of the signal components. Each signal component may be characterized at the receiver by a complex amplitude, a delay, corresponding to a ToA, and an AoA, for example, depending on the available receiver hardware. An outer stage keeps track of the estimates from the inner stage using parallel Kalman filters. Each Kalman filter holds a hypothesis for the number of signal components. Hence, the outer stage also estimates the number of signal components. Although KEST estimates the parameters of signal components jointly, we assume that these estimates are uncorrelated. This assumption is on the one hand based on the uncorrelated scatterer assumption. On the other hand, if such correlations occur, we expect them to affect the estimates only on a short term basis. Therefore, the KEST estimates are unbiased in the long term.

The estimates from the channel estimator at time instant $k$ are combined in the vector $\boldsymbol{z}_{k}$. Within this paper, we use ToA and AoA estimates. They are used as measurement inputs in the second step of Channel-SLAM to estimate the state of the user and the states of the transmitters. The user state consists 
of their position $\boldsymbol{p}_{\mathrm{u}, k}$ and velocity $\boldsymbol{v}_{\mathrm{u}, k}$. At time instant $k$, the user state vector $\boldsymbol{x}_{\mathrm{u}, k}$ is

$$
\boldsymbol{x}_{\mathrm{u}, k}=\left[\begin{array}{ll}
\boldsymbol{p}_{\mathrm{u}, k}^{T} & \boldsymbol{v}_{\mathrm{u}, k}^{T}
\end{array}\right]^{T}=\left[\begin{array}{llll}
x_{k} & y_{k} & v_{x, k} & v_{y, k}
\end{array}\right]^{T} .
$$

As transmitters are considered static, their state at time instant $k$ comprises a location $\boldsymbol{p}_{\mathrm{TX}, k}$ and a delay offset $\tau_{0, k}$. A delay offset can be interpreted as clock offset or synchronization bias towards the user. Channel-SLAM covers both physical and virtual transmitters by the same model and does therefore not require differentiating between the LoS component and MPCs of a received signal: each received signal component is regarded as a LoS component from a transmitter. The state of the $j^{\text {th }}$ transmitter at time instant $k$ is

$$
\boldsymbol{x}_{\mathrm{TX}, k}^{<j>}=\left[\begin{array}{ll}
\boldsymbol{p}_{\mathrm{TX}, k}^{<j>T} & \tau_{0, k}^{<j>}
\end{array}\right]^{T}=\left[\begin{array}{lll}
x_{\mathrm{TX}, k}^{<j>} & y_{\mathrm{TX}, k}^{<j>} & \tau_{0, k}^{<j>}
\end{array}\right]^{T} .
$$

The full state vector is

$$
\begin{aligned}
\boldsymbol{x}_{k} & =\left[\begin{array}{llll}
\boldsymbol{x}_{\mathrm{u}, k}^{T} & \boldsymbol{x}_{\mathrm{TX}, k}^{<1>T} & \ldots & \boldsymbol{x}_{\mathrm{TX}, k}^{<N_{\mathrm{TX}, \mathrm{k}}}>^{T}
\end{array}\right]^{T} \\
& =\left[\begin{array}{ll}
\boldsymbol{x}_{\mathrm{u}, k}^{T} & \left.\boldsymbol{x}_{\mathrm{TX}, k}\right]^{T}
\end{array}\right]^{T}
\end{aligned}
$$

where $N_{\mathrm{TX}, \mathrm{k}}$ is the number of transmitters detected at time instant $k$.

In Channel-SLAM, the state vector in Eq. (4) is estimated with Bayesian recursive estimation. In particular, a RaoBlackwellized particle filter [12], [13] is implemented [7]. Generally, in a particle filter, the involved probability density functions (PDFs) in Bayesian recursive estimation are represented by a set of samples in the state space. These samples are called particles, and each of the particles is assigned a weight. A particle can be regarded as one hypothesis for the true state. The structure of the Rao-Blackwellized particle filter in Channel-SLAM is such that a user particle filter estimates the user state. The states of the transmitters are estimated with a particle filter for each user particle independently from the other user particles. Mathematically, the posterior PDF for the history of the state vector $\boldsymbol{x}_{0: k}$ from time instants zero to $k$ is factorized by

$$
\begin{aligned}
\mathrm{p}\left(\boldsymbol{x}_{0: k} \mid \boldsymbol{z}_{\mathrm{R}, 1: k}, \boldsymbol{u}_{1: k}\right)= & \mathrm{p}\left(\boldsymbol{x}_{\mathrm{TX}, 0: k}, \boldsymbol{x}_{\mathrm{u}, 0: k} \mid \boldsymbol{z}_{\mathrm{R}, 1: k}, \boldsymbol{u}_{1: k}\right) \\
= & \mathrm{p}\left(\boldsymbol{x}_{\mathrm{u}, 0: k} \mid \boldsymbol{z}_{\mathrm{R}, 1: k}, \boldsymbol{u}_{1: k}\right) \\
& \times \mathrm{p}\left(\boldsymbol{x}_{\mathrm{TX}, 0: k} \mid \boldsymbol{x}_{\mathrm{u}, 0: k}, \boldsymbol{z}_{\mathrm{R}, 1: k}\right),
\end{aligned}
$$

where $\boldsymbol{u}_{1: k}$ is a control input, $\boldsymbol{z}_{\mathrm{R}, 1: k}$ are the measurements, i.e., estimates from the channel estimator, for time instants one to $k$. With the control input, additional sensors such as from a gyroscope may be incorporated. The first factor in the second line of Eq. (5) is the posterior PDF of the user state. Following the idea of the particle filter, it is represented by

$$
\mathrm{p}\left(\boldsymbol{x}_{\mathrm{u}, 0: k} \mid \boldsymbol{z}_{1: k}, \boldsymbol{u}_{1: k}\right)=\sum_{i=1}^{N_{p}} w_{0: k}^{<i>} \delta\left(\boldsymbol{x}_{\mathrm{u}, 0: k}-\boldsymbol{x}_{\mathrm{u}, 0: k}^{<i>}\right),
$$

with $\boldsymbol{x}_{\mathrm{u}, 0: k}^{<i>}$ being a particle in the user particle filter, also called user particle, $w_{0: k}^{<i>}$ its associated weight, and $N_{p}$ the number of user particles.
The states of the transmitters can be estimated independently from each other for each user particle, as the estimates from KEST are assumed uncorrelated. Hence, for each particle in the in user particle filter, there is one particle filter for each transmitter estimating the transmitter's state. The posterior PDF of the history $\boldsymbol{x}_{\mathrm{TX}, 0: k}^{<i, j>}$ of the $j^{\text {th }}$ transmitter's state for the $i^{\text {th }}$ user particle from time instants zero to $k$ is factorized by

$$
\mathrm{p}\left(\boldsymbol{x}_{\mathrm{TX}, 0: k}^{<i>} \mid \boldsymbol{x}_{\mathrm{u}, 0: k}^{<i>}, \boldsymbol{z}_{\mathrm{R}, 1: k}\right)=\prod_{j=1}^{N_{\mathrm{TX}, \mathrm{k}}} \mathrm{p}\left(\boldsymbol{x}_{\mathrm{TX}, 0: k}^{<i, j>} \mid \boldsymbol{x}_{\mathrm{u}, 0: k}^{<i>}, \boldsymbol{z}_{\mathrm{R}, 1: k}\right),
$$

where the number of transmitters $N_{\mathrm{TX}, \mathrm{k}}$ corresponds to the number of signal components detected by the channel estimator. The $\ell^{\text {th }}$ of the $N_{p, \text { Tx }}$ particles of the $j^{\text {th }}$ transmitter of the $i^{\text {th }}$ user particle is denoted by $\boldsymbol{x}_{\mathrm{TX}, 0: k}^{<i, j,}$, and its associated weight by $w_{0: k}^{<i, j, \ell>}$. The posterior PDF of the $j^{\text {th }}$ transmitter for the $i^{\text {th }}$ user particle is represented by

$$
\begin{aligned}
\mathrm{p} & \left(\boldsymbol{x}_{\mathrm{TX}, 0: k}^{<i, j>} \mid \boldsymbol{z}_{1: k}, \boldsymbol{x}_{\mathrm{u}, 0: k}^{<i>}\right) \\
= & \sum_{\ell=1}^{N_{p, \mathrm{Tx}}} w_{0: k}^{<i, j, \ell>} \delta\left(\boldsymbol{x}_{\mathrm{TX}, 0: k}^{<i, j>}-\boldsymbol{x}_{\mathrm{TX}, 0: k}^{<i, j, \ell>}\right) .
\end{aligned}
$$

The number of transmitter particles $N_{p, \text { Tx }}$ may differ for different time instants, user particles and transmitters. For the sake of notational brevity, the corresponding indices are omitted in $N_{p, \text { Tx }}$.

Tracking the signal components' parameters with filters as done in KEST inherently yields associations of the signal components from one time instant to another. Following the idea of multipath assisted positioning, it yields associations between signals and transmitters at consecutive time steps.

\section{VISIBILITY MAPPING}

As a user travels through a scenario, a LoS signal from a transmitter may be received or not. If such a signal is received, we say that a transmitter is visible.

We propose to store information on transmitter visibilities in a location-based hexagonal grid map. The two-dimensional space is divided into adjoining hexagons. In one hexagon, a transmitter may be visible at one time, and not visible another time. There are multiple possible reasons for that. The first reason corresponds to a discretization error. A transmitter might be visible from one part of the hexagon, but not from another part, due to the geometry of the environment. The larger the size of the hexagon, the more likely is such a case. The second reason stems from the channel estimator in the first step of Channel-SLAM. It might track a signal component one time and not track it another time for the same or a very close user location as before, although the corresponding transmitter is in LoS. Such a behavior may occur if the received power of the signal component is near the receiver sensitivity, resulting in a missed detection. In addition, two or more signal components may be close to each other in terms of tracked parameters, such as ToA or AoA. Thus, they may arrive at the receiver 
from similar angles almost simultaneously. In such a case, the channel estimator may mistake the two signal components for only one, as it is not able to resolve them. The smaller the signal bandwidth, the more likely is such a case. A third reason for changing visibilities of transmitters is a change in the environment. Objects or people may temporarily block or shadow signal components, or cause additional MPCs. Further reasons include a wrong data association or a biased user position estimate.

To map information on visibilities, the user records at every time instant $k$ which transmitters are visible and which are not from the hexagon they are currently in. If the $j^{\text {th }}$ transmitter is visible in the $h^{\text {th }}$ hexagon, at time instant $k$, the counter $C_{h, k}^{<j>}$ is increased by one. Otherwise, the counter $\bar{C}_{h, k}^{<j>}$ is increased by one. The counters $C_{h, k}^{<j>}$ and $\bar{C}_{h, k}^{<j>}$ store how often a transmitter has been visible or not within a hexagon from time instants zero to $k$. The information whether a transmitter is visible or not at a time instant is obtained from the outer stage of the KEST estimator.

If only one such observation is available in a hexagon, say the user has been in the hexagon only once when a certain set of transmitters was visible, the estimate that these transmitters are always visible purely based on that one observation would be presumptuously confident. Therefore, we model the belief that a transmitter is visible from within a hexagon with a Beta distribution. A Beta distribution can incorporate prior knowledge to prevent overconfident estimates on probabilities if only few observations and thus unreliable information are available. If no prior knowledge on visibilities is available, as is in general the case, the prior visibility probabilities are assumed uniform. The PDF of a Beta function with parameters $p$ and $q$ is defined as

$$
\mathbb{B}(x ; p, q)=\frac{1}{\mathrm{~B}(p, q)} x^{p}(1-x)^{q}
$$

for $x$ the interval $(0,1)$, and $\mathbb{B}(x ; p, q)=0$ otherwise. The function $\mathrm{B}(p, q)$ is the Beta function [14].

Observations on the visibilities of a transmitter can be regarded as the realization of a binomially distributed random variable. The Beta distribution is the conjugate prior of the binomial distribution. Hence, over time, as more and more observations on the visibility of a transmitter are made in a hexagon, the parameters of the Beta distribution are updated by these observations, and the expectation value of the belief that a transmitter is visible or not gets closer to the actual probability. The parameters of the Beta distribution representing the belief at time instant $k$ that the $j^{\text {th }}$ transmitter in the $h^{\text {th }}$ hexagon is visible are

$$
\begin{aligned}
& p_{h}^{<j>}=C_{h, k}^{<j>}+\nu_{h}^{<j>} \text { and } \\
& q_{h}^{<j>}=\bar{C}_{h, k}^{<j>}+\bar{\nu}_{h}^{<j>} .
\end{aligned}
$$

With the scalars $\nu_{h}^{<j>}$ and $\bar{\nu}_{h}^{<j>}$, prior knowledge on the transmitter visibility may be incorporated. For notational brevity, the time index $k$ is omitted in $p_{h}^{<j>}$ and $q_{h}^{<j>}$.

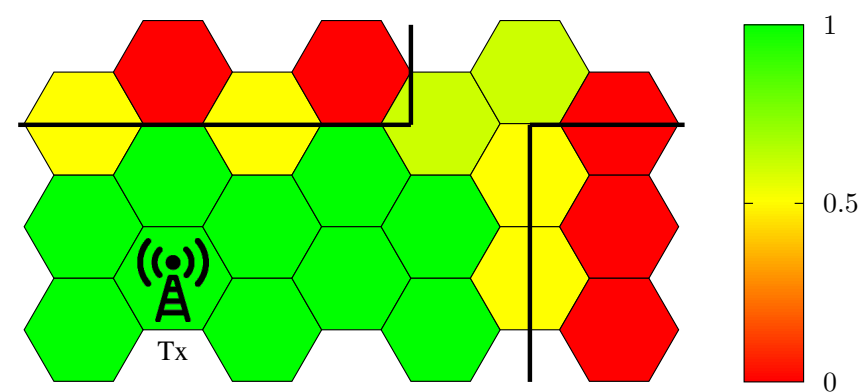

Fig. 2. The hexagonal visibility map is exemplarily plotted for the transmitter Tx. The black lines are walls that reflect the transmit signal. The colors of the hexagons indicate the probabilities that a transmitter is visible for the user from an arbitrary point within the hexagon.

The actual probability that the $j^{\text {th }}$ transmitter is visible from an arbitrary location in hexagon $h$ is in general unknown. We denote the random variable for this probability by $\boldsymbol{V}_{h}^{<j>}$. The set of the random variables for the $N_{\text {TX,k }}^{\mathrm{a}}$ transmitters' visibilities is denoted by

$$
\boldsymbol{M}=\left\{\left\{\boldsymbol{V}_{h}^{<1>}, \ldots, \boldsymbol{V}_{h}^{<N_{\mathrm{TX}, \mathrm{k}}^{\mathrm{a}}>}\right\}^{h=1, \ldots, N_{H}}\right\},
$$

which we call a visibility map. The number $N_{\text {TX,k }}^{\mathrm{a}}$ is the overall number of transmitters that have been detected by the channel estimator throughout the user trajectory up to time instant $k$. Likewise, the overall number of hexagons that a user has visited is denoted by $N_{H}$. Although this number of hexagons may grow over time, the time index in $N_{H}$ is omitted.

Fig. 2 illustrates a hexagonal visibility map for one transmitter Tx. In the scenario, there are four black lines that represent walls reflecting the transmit signal. The color a hexagon is filled represents the probability that the transmitter Tx is visible from an arbitrary position within this hexagon. For example, in the yellow hexagons, this probability of the transmitter being visible is 0.5 , corresponding to the probability that the user is in front or behind a wall.

\section{MAP MATCHING}

When maps are exchanged among users, the unknown relative rotation $\beta$ and translation $\bar{x}$ and $\bar{y}$ between the coordinate systems of the map and the user receiving the map need to be estimated. A map consists of the estimates, i.e., PDFs, of transmitter states and the visibility map as described in Section III. We denote a map that is transmitted by the term prior map, and the transmitter states and visibilities estimated by the user receiving the prior map by user map. In addition to the transformation parameters, the correspondences of transmitters in the user map and the prior map need to be found. Estimating such correspondences in and the transformation parameters between two maps is denoted by the term map matching.

\section{A. Map Matching Based on Transmitter Locations}

We have previously presented a map matching algorithm that is based purely on the transmitter's locations in the two maps [10], which is briefly summarized in the following. 
The absolute locations of transmitters in the two maps have no informative value without the rotation and translation parameters relating the coordinate systems of the user and the prior map. However, the relative distances among transmitters may be used to obtain a map match. Given a set of $N_{T}$ transmitters in the prior map and a set of $N_{T}$ transmitters in the user map, the best correspondences among the transmitters are obtained in a least squares (LS) sense regarding these relative distances. Once the correspondences are known, the transformation parameters can be calculated with an LS approach.

Based on the underlying user trajectories of the user map and the prior map, though, certain transmitters do have an actual correspondence in the respective other map, and some others do not. The above two steps would therefore have to be performed for all possible subsets of $N_{T}$ transmitters in the two maps. Instead, we apply a variant of the RANSAC algorithm. In each iteration of our adapted RANSAC, $N_{T}$ transmitters from both maps are chosen randomly, where $N_{T}$ is the minimum number of transmitters to calculate a unique map match solution. For the one rotation and two translation parameters, $N_{T}=3$. Based on these transmitters, the best map match is calculated based on LS. With this map match and the corresponding transformation parameters, further correspondences of transmitters are sought. In particular, the prior map is transformed into the coordinate system of the user. If the distance between a transformed transmitter in the prior map and a transmitter in the user map is below a threshold $\delta_{d}$, this pair is added to the set of correspondences.

The RANSAC algorithm chooses a set of $N_{T}$ transmitters repeatedly for a number of times. The best map match is chosen based on an error term $E_{\mathrm{C}}$ for each possible map match returned by RANSAC. The error term includes on the one hand the distances between corresponding transmitters in the user and the prior map. On the other hand, the number of correspondences is included as well to account for the fact that the more correspondences are found, the more reliable the map match is considered.

\section{B. Map Matching with Visibilities}

Yet, in scenarios with certain geometries, ambiguities in map matching arise, in particular if only few transmitters can be used to find a map match. Such a case is drawn as an example in Fig. 3 In Fig. 3 (a), the green circles represent transmitter location estimates for a prior map, and the red squares are estimates for transmitter locations of a user map. Fig. 3 (b) shows a possible map match where a correspondence for three transmitter pairs was found. The dashed red circles around the user map transmitters are of radius $\delta_{d}$. If a transmitter from the prior map falls within such a circle after the transformation, a correspondence between the two transmitter has been found. In Fig. 3 (c), only two correspondences are found, whereas in Fig. 3 (d), its again three transmitter correspondences. There is now an ambiguity between the map matching solutions in Fig. 3 (b) and Fig. 3

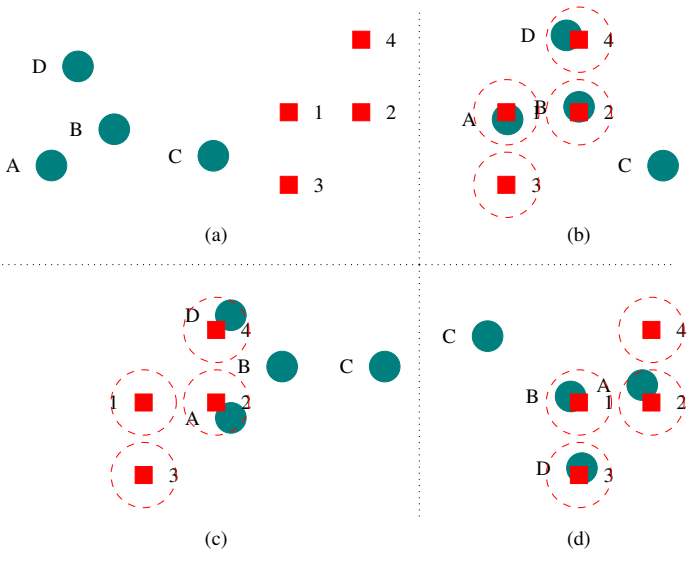

Fig. 3. In (a), the green circles represent are transmitter locations from the prior map, and the red squares transmitter locations from the user map. In (b)-(d), different map matching solutions with three (b), two (c), and again three (d) correspondences are found.

(d), which result in a similar map matching error but are very different in terms of rotation and translation parameters.

The problem of map matching purely based on transmitter locations is a lack of diversity among the transmitters. Thus, we propose to increase their diversity by including visibility maps as described in Section III in the map matching process.

The RANSAC algorithm we use for map matching returns a number $N_{\text {hyp }}$ of hypotheses for a map match. Each hypothesis includes the rotation and translation parameters and the correspondence set $\mathrm{C}_{s}$, where $s$ is the hypothesis index. The correspondence set consists of tuples $\left(j_{\mathrm{u}}, j_{\mathrm{p}}\right)$, where $j_{\mathrm{u}}$ is the index of a transmitter in the user map, and $j_{\mathrm{p}}$ the index of the corresponding transmitter in the prior map. The cardinality of $\mathrm{C}_{s}$ is donated by $N_{\mathrm{C}, s}$. Each hypothesis comes with a map matching error $E_{\mathrm{C}, s}$ that indicates the goodness of a map match. The error is calculated as

$$
E_{\mathrm{C}, s}=\frac{1}{N_{\mathrm{C}, s}} \sum_{\left(j_{\mathrm{u}}, j_{\mathrm{p}}\right) \in \mathrm{C}_{s}}\left\|\boldsymbol{\mu}_{j_{\mathrm{u}}}^{u}-\tilde{\boldsymbol{\mu}}_{j_{\mathrm{p}}}^{p}\right\|-N_{\mathrm{C}, s} \rho_{\mathrm{rew}},
$$

where $\boldsymbol{\mu}_{j_{\mathrm{u}}}^{u}$ is the mean of the $j_{\mathrm{u}}^{\text {th }}$ transmitter in the user map, and $\tilde{\boldsymbol{\mu}}_{j_{\mathrm{p}}}^{p}$ the mean of the $j_{\mathrm{p}}{ }^{\text {th }}$ transmitter after the rotation and translation. The term $\rho_{\text {rew }}$ is a reward term that rewards hypotheses with large correspondence sets.

For map matching, only transmitters with a small enough variance are used, since a high uncertainty about the transmitter locations leads to a high uncertainty about the map match. Hence, the Euclidean distance between the means of the transmitter location estimates is used as a distance metric in Eq. 12.

The $s^{\text {th }}$ hypothesis from RANSAC is denoted by $H_{s}$. To decrease the computational complexity and to reject very unlikely map matching hypotheses, we consider only hypotheses whose error $E_{\mathrm{C}, s}$ does not exceed a threshold $\tau_{\mathrm{E}}$.

For a given map match hypothesis, the visibility map in the prior map needs to be transformed with the estimated parameters into the underlying hexagonal grid of the user's 


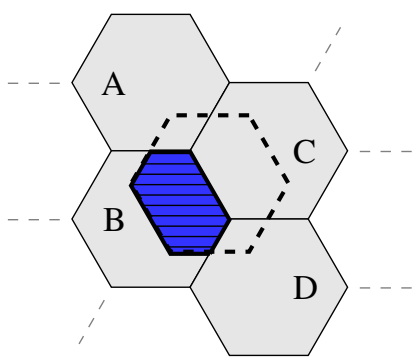

Fig. 4. The hexagons A, B, C and D are in the equivalent prior map, which is in the user's hexagonal grid. The visibility infomation from the rotated and translated hexagon from the prior map indicated by the thick dashed lines is taken into the hexagons of the equivalent map considering the overlapping area of the two respective hexagons. For example, the overlapping are of the prior map hexagon and hexagon B is drawn in blue and hatched, and covers $5 / 12$ of an entire hexagon's area.

visibility map. We name such a transformed map an equivalent prior map. For the transformation, the equivalent prior map is empty in the beginning, i.e., the visibility counters for all transmitters in all hexagons are set to zero. Each hexagon in the prior map is transformed with the estimated rotation and translation parameters if the hypothesis. The visibility counters of a transformed hexagon are added for all transmitters to the underlying hexagons in the equivalent prior map considering the overlapping ratios. An example is depicted in Fig. 4. A hexagon from the prior map indicated by the thick dashed edges is rotated and translated. This transformed hexagon lies now on top of the equivalent prior map that is aligned with the hexagonal grid of the user's visibility map. The overlapping area of the transformed hexagon with hexagon $\mathrm{B}$ from the equivalent hexagonal map, drawn in dark blue and hatched, is $5 / 12$ of the total area of one hexagon. Thus, the visibility counters of hexagon B are increased by the visibility counters of the transformed hexagon multiplied by $5 / 12$. Likewise, the counters of the hexagons $\mathrm{A}, \mathrm{C}$, and $\mathrm{D}$ in the equivalent prior map are increased. The above procedure is performed for every hexagon in the prior map, such that the equivalent prior map holds the information on the original prior map in the user's hexagonal grid. While the hexagons in the user and the prior map are of the same size in Fig. 4, visibility maps of different hexagon sizes can be processed in the same way. The set of indices of hexagons that are in both the user map and the equivalent prior map for the $s^{\text {th }}$ hypothesis is denoted by $\mathrm{H}_{o, s}$.

Once the $N_{\text {hyp }}$ equivalent prior maps are obtained, a likelihood ratio test [15] on the hypotheses is performed to choose the best hypothesis $\hat{H}$ for the map match. It is chosen as

$$
\begin{aligned}
\hat{H} & =\underset{H_{s}, s=1, \ldots, N_{\text {hyp }}}{\arg \max } \mathrm{p}\left(H_{s} \mid \boldsymbol{M}\right) \\
& =\underset{H_{s}, s=1, \ldots, N_{\text {hyp }}}{\arg \max } \mathrm{p}\left(\boldsymbol{M} \mid H_{s}\right) \mathrm{p}\left(H_{s}\right),
\end{aligned}
$$

where the random variable $M$ is the user visibility map. In the second line of Eq. (13), we have used Bayes's theorem, allowing to incorporate prior knowledge of the hypotheses. The prior knowledge is obtained from the map matching error
$E_{\mathrm{C}, s}$ for the $s^{\text {th }}$ hypothesis as

$$
\mathrm{p}\left(H_{s}\right)=\frac{\sum_{\tilde{s}=1}^{N_{\text {hyp }}} E_{\mathrm{C}, \tilde{s}}^{+}}{E_{\mathrm{C}, s}^{+}},
$$

where $E_{\mathrm{C}, s}^{+}=\max \left\{E_{\mathrm{C}, s}, 1 \mathrm{~m}\right\}$. The best hypothesis from Eq. 13. can thus be written as

$$
\hat{H}=\underset{H_{s}, s=1, \ldots, N_{\text {hyp }}}{\arg \max } \frac{\mathrm{p}\left(\boldsymbol{M} \mid H_{s}\right)}{E_{\mathrm{C}, s}^{+}} .
$$

The second term in the argument on the right hand side of Eq. (14) is calculated following Eq. (9) as

$$
\begin{aligned}
\mathrm{p}\left(\boldsymbol{M} \mid H_{s}\right)= & \prod_{\left(j_{\mathrm{u}}, j_{\mathrm{p}}\right) \in \mathrm{C}_{s}} \prod_{h \in \mathrm{H}_{o, s}} \frac{1}{\mathrm{~B}\left(p_{h}^{<j_{\mathrm{u}}>}, q_{h}^{<j_{\mathrm{u}}>}\right)} \\
& \times V_{h}^{<j_{\mathrm{p}}>}{ }^{\left(p_{h}^{<j_{\mathrm{u}}>}-1\right)}\left(1-V_{h}^{<j_{\mathrm{p}}>}\right)^{\left(q_{h}^{<j_{\mathrm{u}}>}-1\right)},
\end{aligned}
$$

where $V_{h}^{<j_{\mathrm{p}}>}$ is the expectation value of the Beta distribution regarding the visibility of the $j_{\mathrm{p}}{ }^{\text {th }}$ transmitter in the $h^{\text {th }}$ hexagon in the equivalent prior map. The parameters $p_{h}^{<j_{\mathrm{u}}>}$ and $q_{h}^{<j_{\mathrm{u}}>}$ are obtained from Eq. 10 referring to the user map. The entire map matching algorithm is summarized in Algorithm 1

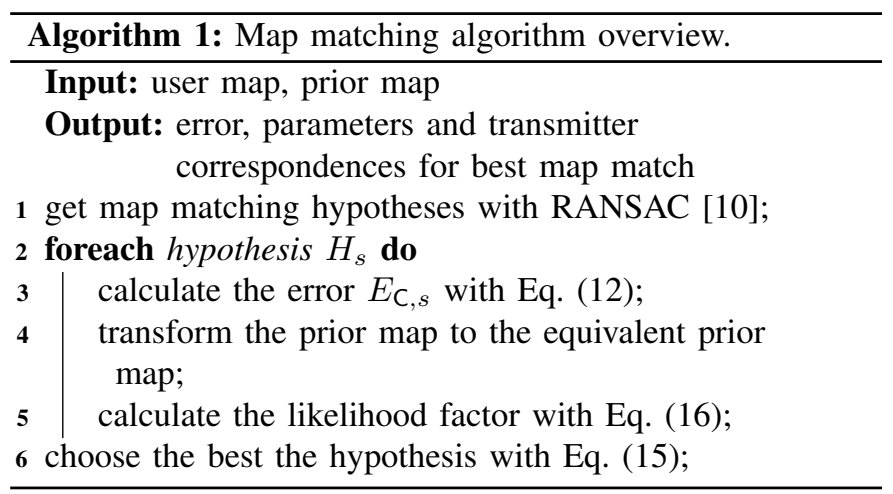

For numerical stability, the arguments in Eq. (15) and the factors in Eq. (16) are calculated in logarithmic domain.

Once a map match has been found, the information in the transformed prior map can be used to improve the positioning performance in Channel-SLAM by establishing associations between signal components and transmitters in the prior map as shown in [16].

\section{Simulations}

\section{A. Simulation Scenario}

To evaluate our approach, we performed simulations in an indoor scenario depicted in Fig. 5, showing the top view of a shopping mall with one physical transmitter depicted by the red triangle labeled Tx. The transmit signal from the physical transmitter is reflected at the thick black lines representing walls, and scattered at the black dots representing point scatterers. There are six user trajectories in the scenario 


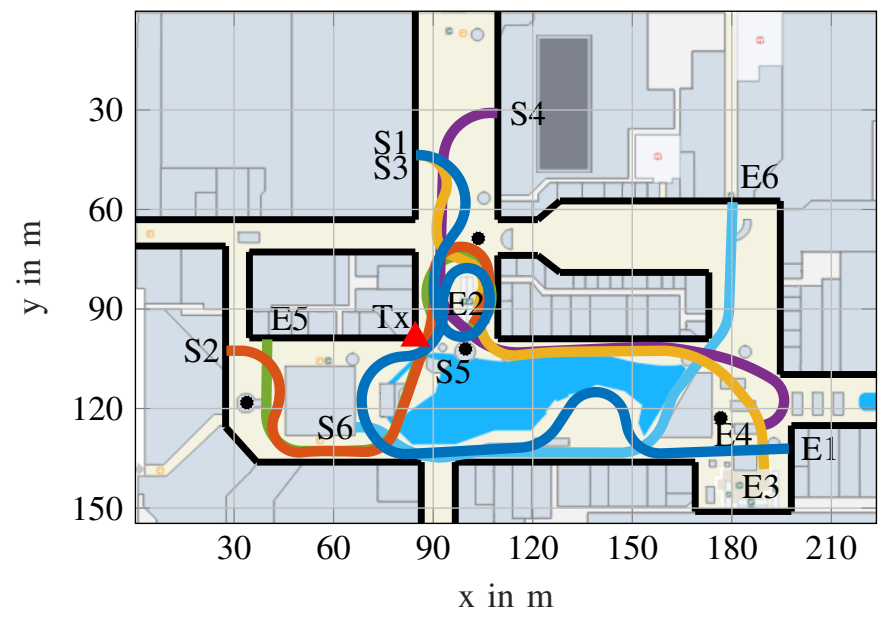

Fig. 5. Overview of the indoor simulation scenario with one physical transmitter labeled Tx. The black lines represent walls and the black dots point scatterers. The different user tracks are drawn with different colors, where the $i^{\text {th }}$ trajectory's start and end points are labeled S $i$ and E $i$, respectively.

plotted in different colors. The start and end points of the $i^{\text {th }}$ trajectory are labeled $\mathrm{S} i$ and $\mathrm{E} i$, respectively.

The physical transmitter continuously transmits a signal of rectangular shape in frequency domain with a bandwidth of $100 \mathrm{MHz}$. It is reflected and scattered in the environment and arrives at the user as a superposition of different signal components. In our simulations, single and double reflections and/or scatterings are taken into account. The average signalto-noise ratios (SNRs) for the six tracks are $13.1 \mathrm{~dB}, 14.9 \mathrm{~dB}$, $10.4 \mathrm{~dB}, 10.1 \mathrm{~dB}, 15.3 \mathrm{~dB}$ and $7.5 \mathrm{~dB}$, respectively.

A user traveling with a constant speed of $1 \mathrm{~m} / \mathrm{s}$ takes a snapshot of the received signal every $100 \mathrm{~ms}$. In our simulations, we create the true channel impulse response (CIR) at each user position with ray-tracing, limit it to a bandwidth of $100 \mathrm{MHz}$ and add white Gaussian noise (WGN) to obtain a snapshot of the received signal. The recorded snapshot is then used for Channel-SLAM by a user. We assume that a user is equipped with an antenna array and uses ToA and AoA estimates.

The side length of the hexagons in a visibility map is set to $2 \mathrm{~m}$.

The users do not know the locations of the physical transmitter, the walls and scatterers, or the virtual transmitters. Their starting location is unknown as well, and they define their local coordinate systems arbitrarily.

\section{B. Evaluations}

We define the first track depicted in dark blue with start point $\mathrm{S} 1$ and end point E1 as reference track. A user travels along the reference track doing Channel-SLAM without a prior map. Afterward, the user hands their estimated map of transmitter states and visibilities as a prior map to the other five users who then travel along the five other tracks. Walking along their track, each user tries to find a map match with and without using visibility information. If no visibility information is used, the best map match is calculated as in [10] purely based on transmitter locations following Eq. (12).
TABLE I

MAP MATCHING RESULTS WITH VISIBILITY INFORMATION

\begin{tabular}{c||c|c|c||c} 
& better & worse & same & sum \\
\hline Track 2 & 65 & 8 & 527 & 600 \\
Track 3 & 45 & 8 & 547 & 600 \\
Track 4 & 48 & 15 & 537 & 600 \\
Track 5 & 32 & 7 & 561 & 600 \\
Track 6 & 109 & 11 & 480 & 600 \\
\hline
\end{tabular}

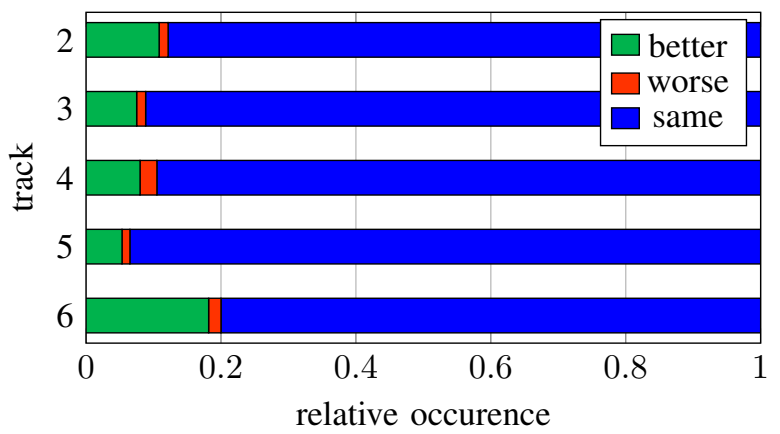

Fig. 6. For each track, the normalized numbers of occurrences are plotted for the three cases of a better, worse, or the same map match with using visibility maps.

To evaluate our approach, all true virtual transmitter locations of order one and two are calculated based on the scenario. For each user, the true and estimated transformation parameters are compared. For map matching with and without exploiting the visibility map, we regard three possible outcomes. In the first case, the transformation parameters are the same. In the second case, they are in some sense better, and in the third case they are worse.

The first case means that using the visibility map does not influence the map matching outcome. In the second case, the visibility map has indeed improved the map match due to the likelihood ratio test. In the third case, this likelihood ratio test decreases the goodness of the map match.

The locations of the transmitters in the two spatial dimensions have a certain mean and a covariance, which we use to define a Gaussian distribution. This distribution of the transmitter locations after the transformation with the true parameters serves as the reference distribution. If the parameters of two map matches are different with and without using visibility maps, the map match with a smaller Kullback-Leibler divergence (KLD) between the corresponding Gaussian distribution and the reference distribution is considered the better one.

We performed 600 runs of Channel-SLAM with map matching for every of the five tracks. The results in terms of whether a map match was the same, better or worse when using visibility maps or not are given in Table II, and the relative occurrences for the three cases are plotted in Fig. 6

The sixth track drawn in light blue in Fig. 5 is never close to the region where the reference track makes a loop near the physical transmitter. Thus, the set of virtual transmitters that 
both the sixth user and the user going along the reference track observe is relatively small, and map matching ambiguities are more likely. This observation is in accordance with Fig. 6 where we see that the map matching results are influenced by visibility information for the sixth track more than for the other tracks.

\section{Discussion}

As can be seen in Fig. 5, the overlaps of the reference track with the other tracks are relatively small. Hence, the contribution of visibility information for map matching is limited, since relatively few hexagons are taken into account. Nevertheless, we observe in the simulation data, that when map matching ambiguities occur, i.e., when different transformation parameters seem likely given the locations of transmitters, visibility information can help resolve these ambiguities. In the long run, we want users to share transmitter maps in a crowdsourcing scheme. Then, a prior map covers a larger area and more hexagons in the user map and the prior map overlap. We expect more improvement in the robustness in the map matching results in terms of less cases where the information on visibility of transmitters leads to a worse map match.

In addition, visibility maps considerably improve the positioning performance when they are used for data association as shown in [17]. Thus, the memory and transmission overhead of visibility maps in addition to transmitter state information is justified, as visibility maps can be used for both data association and map matching.

Map matching can be computationally expensive if there are many transmitters in the user and in the prior map, as typically a high number of different map matching hypotheses are found and need to be tested regarding their likelihood. However, the user map, i.e., the state and visibility estimates for the transmitters estimated by the user, is not expected to change a lot from one time instant to another. Thus, the result of map matching is likely to stay the same during such a short time interval, and it does not need to be performed at every time instant. In our implementations, a new map match is calculated after every meter the user has traveled, or when the uncertainty about a transmitter in the user map has decreased considerably in between. In addition, map matching can be performed in parallel to the actual Channel-SLAM algorithm.

\section{CONCLUSION}

A robust map matching scheme is a key enabler for cooperative Channel-SLAM. Since virtual transmitters arise only due to reflections and scattering in the environment, they lack diversity, which makes map matching a challenging problem, in particular in situations when only few transmitters have a correspondence in the respective other map.

Within this paper, we have investigated how mapping visibilities can improve the robustness and accuracy of map matching in Channel-SLAM. We have derived a map matching algorithm incorporating both the estimated locations of transmitters in the user map and the prior map, and the corresponding visibility maps. The novelty of our new algorithm is to exploit visibilities of transmitters to increase the by nature limited diversity of in particular virtual transmitters. We have increased the robustness in our map matching algorithm if a user had received a map from only one reference user. In scenarios where many more user contribute to a map with more overlaps of the user tracks, we expect the robustness to increase even more.

\section{ACKNOWLEDGEMENT}

This work was partially supported by the DLR project Navigation 4.0.

\section{REFERENCES}

[1] Y. Shen and M. Win, "On the Use of Multipath Geometry for Wideband Cooperative Localization," in IEEE Global Telecommunications Conference (GLOBECOM), Nov. 2009, pp. 1-6.

[2] P. Meissner and K. Witrisal, "Multipath-assisted single-anchor indoor localization in an office environment," in 19th International Conference on Systems, Signals and Image Processing (IWSSIP), Apr. 2012, pp. $22-25$.

[3] A. O'Connor, P. Setlur, and N. Devroye, "Single-sensor RF emitter localization based on multipath exploitation," IEEE Trans. Aerosp. Electron. Syst., vol. 51, no. 3, pp. 1635-1651, Jul. 2015.

[4] E. Leitinger, S. Grebien, and K. Witrisal, "Multipath-Based SLAM Exploiting AoA and Amplitude Information," in IEEE International Conference on Communications Workshops (ICC Workshops), May 2019, pp. 1-7.

[5] R. Mendrzik, H. Wymeersch, G. Bauch, and Z. Abu-Shaban, "Harnessing NLOS Components for Position and Orientation Estimation in 5G Millimeter Wave MIMO,’ IEEE Trans. Wireless Commun., vol. 18, no. 1, pp. 93-107, Jan. 2019.

[6] C. Gentner, T. Jost, and A. Dammann, "Indoor Positioning using Time Difference of Arrival between Multipath Components," in IEEE IPIN, Montbeliard, France, Oct. 2013.

[7] C. Gentner, T. Jost, W. Wang, S. Zhang, A. Dammann, and U.-C. Fiebig, "Multipath Assisted Positioning with Simultaneous Localization and Mapping," IEEE Trans. Wireless Commun., vol. 15, no. 9, pp. 6104 6117, Sep. 2016

[8] H. Durrant-Whyte and T. Bailey, "Simultaneous localization and mapping: part I," IEEE Robot. Autom. Mag., vol. 13, no. 2, pp. 99-110, Jun. 2006.

[9] M. Ulmschneider, D. C. Luz, and C. Gentner, "Exchanging Transmitter Maps in Multipath Assisted Positioning," in IEEE/ION Position, Location and Navigation Symposium (PLANS), 2018.

[10] M. Ulmschneider and C. Gentner, "RANSAC for Exchanging Maps in Multipath Assisted Positioning," in IEEE International Conference on Industrial Cyber Physical Systems (ICPS), 2019.

[11] M. A. Fischler and R. C. Bolles, "Random Sample Consensus: A Paradigm for Model Fitting with Applications To Image Analysis and Automated Cartography," Commun. ACM, vol. 24, no. 6, pp. 381-395, Jun. 1981.

[12] M. Arulampalam, S. Maskell, N. Gordon, and T. Clapp, "A Tutorial on Particle Filters for Online Nonlinear/non-Gaussian Bayesian Tracking,' IEEE Trans. Signal Process., vol. 50, no. 2, pp. 174-188, Feb. 2002.

[13] A. Doucet, S. Godsill, and C. Andrieu, "On sequential Monte Carlo sampling methods for Bayesian filtering," Statistics and Computing, vol. 10, no. 3, pp. 197-208, Jul. 2000.

[14] G. Andrews, R. Askey, and R. Roy, Special Functions, ser. Encyclopedia of Mathematics and its Applications. Cambridge University Press, 1999.

[15] T. M. Cover and J. A. Thomas, Elements of Information Theory, ser. Wiley Series in Telecommunications and Signal Processing. New York, NY, USA: Wiley-Interscience, 2006.

[16] M. Ulmschneider, C. Gentner, T. Jost, and A. Dammann, "Multiple Hypothesis Data Association for Multipath-Assisted Positioning," in 14th Workshop on Positioning, Navigation and Communications (WPNC), Oct. 2017.

[17] M. Ulmschneider, C. Gentner, and A. Dammann, "Data Association among Physical and Virtual Radio Transmitters with Visibility Regions," in IEEE 90th Vehicular Technology Conference (VTC2019-Fall), Sep. 2019. 